\title{
Multiple Sklerose
}

\section{Bald neue Behandllungschancen bei früh aktiver MS?}

Eei einer schubförmigen Multiplen Sklerose (MS) mit initial hoher Krankheitsaktivität oder bei einer hohen Aktivität unter immunmodulierender Therapie ist die klinische Prüfung neuer Wirkstoffe gegen Placebo nicht möglich. Daher erfolgt in diesem Falle ein Head-toHead-Vergleich mit einer aktiven Kontrollsubstanz.

Eine Phase-II-Studie zeigte eine deutlich höhere Wirksamkeit des humanisierten monoklonalen Antikörpers Alemtuzumab in einer Dosis von $12 \mathrm{mg}$ im Vergleich zu $44 \mu \mathrm{g}$ Interferon(IFN) $\beta$-1a (s.c.) bei Patienten mit früher aktiver MS [1]. Wie Professor Alasdair Coles, Neurologe der Universität Cambridge/UK, berichtete, senkte Alemtuzumab in dieser Studie die Schubrate gegenüber der aktiven Kontrollsubstanz signifikant um $67 \%(p<0,001), 76 \%$ der Patienten blieben über drei Jahre schubfrei, in der Kontrollgruppe nur $50 \%$.

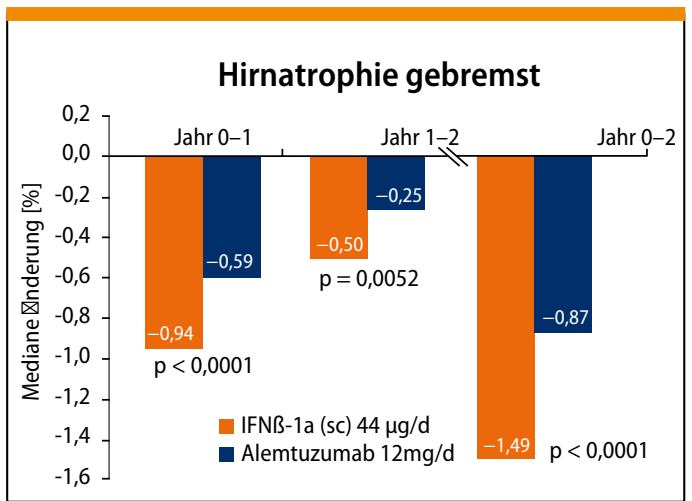

Abbildung 1 Im zweiten Jahr der Antikörpertherapie war die Hirnatrophie auf das Normalmaß reduziert (nach [2]).
Ohne Behinderungsprogression blieben $92 \%$ versus $73 \%$. Die hohe Wirksamkeit hielt auch über fünf Jahre an, ergänzte Coles. Dabei waren unter Alemtuzumab milde bis mittelschwere Infusionsreaktionen häufig, die aber laut Coles gut zu beherrschen waren. Die Alemtuzumab-Therapie schien nicht mit opportunistischen Infektionen assoziiert zu sein. Allerdings wurde bei einigen Patienten eine Antikörper-abhängige Autoimmunität festgestellt, weshalb dieser Aspekt in weiteren Studien besonders kontrolliert werden soll.

Die randomisierte Phase-III-Studie CARE-MS I bestätigte die hohe Wirksamkeit des Antikörpers im Vergleich zu $44 \mu \mathrm{g}$ IFN $\beta$-1a (s.c.) bei 581 Patienten mit früher aktiver MS [2]. Über zwei Jahre senkte die Therapie mit Alemtuzumab die jährliche Schubrate signifikant um $55 \%$ stärker als die Behandlung mit dem IFN $\beta$ $(0,39$ versus 0,18$)$. Auch der Anteil der Patienten mit einer Behinderungsprogression nach EDSS war in der Alemtuzumab-Gruppe mit $8 \%$ geringer als in der IFN $\beta$ Gruppe (11\%). Bemerkenswert fand Coles, dass die Abnahme des Hirnparenchyms im zweiten Jahr in der Alemtuzumab-Gruppe mit - $0,25 \%$ dem entspricht, was bei gesunden Erwachsenen zu erwarten ist (Abbildung 1). Er wertete dies als einen Hinweis auf eine neuroprotektive Wirksamkeit des Antikörpers. Friederike Klein

1. CAMMS223 Trial Investigators. N Engl J Med. 2008; 359: 1786-801

2. Coles A et al., ECTRIMS/ACTRIMS 2011, Amsterdam, Poster P 151

Satellitensymposium "Evolving treatment expectations in MS: Where are we going" ECTRIMS/ACTRIMS 2011, Amsterdam, 21.10.2011 Veranstalter: Genzyme, München

\section{Stipendien für MS-Forschung}

Das Unternehmen Almirall hat die Stipendien 2012 für Forschung auf dem Gebiet der MS-induzierten Spastik an Dr. T. Schultheiß, Zentrum für klinische Neurowissenschaften der Klinik für Neurologie des Uniklinikums Dresden, und an M. C. Molina Díaz, Reha-Abteilung der Universitätsklinik Nuestra señora de la Candelaria University Hospital (Santa Cruz de Tenerife, Spanien) vergeben. Schultheiß untersuchte die Wirksamkeit des Endocannabinoid-System-Modulators Sativex ${ }^{\circledast}$ bei Gangparametern, Müdigkeit und körperlichem Befinden. Díaz hat sich mit dem Nutzen einer Physiotherapie mittels Videokonferenzen bei Patienten mit MS-induzierter Spastik befasst. Den Forschungsprojekten werden $24.000 €$ jährlich zur Verfügung gestellt. Almirall

\section{Quetiapin von neuraxpharm}

Das atypische Antipsychotikum Quetiapin ist ab sofort auch als Quetiapinneuraxpharm ${ }^{\circledast}$ erhältlich. Das Produkt besitzt ein breit gefächertes Indikationsspektrum und bietet den Vorteil einer zusätzlichen Wirkstärke (150 mg). Weiterer Vorteil sind teilbare Filmtabletten bei den Wirkstärken: 100, 200 und 300 mg.

neuraxpharm

\section{Neue Parkinson-Broschüren}

Mit zwei neuen Broschüren erweitert die TEVA Pharma GmbH das Service-Programm „Leben mit Parkinson". Die Hefte "Schlucken \& Parkinson" und "Sprechen \& Parkinson" fassen auf je 20 Seiten in patientengerechter Weise das Wichtigste in Kürze zusammen. Neurologen können sie beim Arzt-Patienten-Gespräch einsetzen und dem Patienten und seinen Angehörigen als einführende Lektüre an die Hand geben. Die kostenlosen Broschüren sind über den Außendienst erhältlich.

$$
\text { Teva }
$$

\section{Webseite zur Alzheimer-Demenz}

Auf der kürzlich aktualisierten Internetseite www.alzheimer-kompakt.de finden Betroffene und Angehörige Informationen, die sie im Alltag mit der Erkrankung Alzheimer-Demenz unterstützen. Die Seite enthält umfangreiches Servicematerial wie Videos, die Tipps im Umgang mit der Erkrankung geben, Broschüren zum Download und eine Suchfunktion, über die User eine Memory-Klinik in der Nähe ihres Wohnortes finden können. Lundbeck 